had been widely expected; with a different development policy this would presumably have been raised. He says of the UKAEA's 1957-58 plan drawn up by a group with a former Treasury planner as chairman, which concentrated development on the advanced gas-cooled reactor with the steam-generating heavy water reactor as a reserve, and ended work on the pressurized water reactor, that it was "well planned to make the best use of available resources, provided that technical economic and political conditions did not change". How does he decide it was well planned? Was the proviso realistic? Or is it an implicit criticism? The resources absorbed in the programme in 20 years with no return at all until 1976 have been enormous. He says much on what went wrong, but gives no comprehensive measure of the vast cost of the

\section{Energy policy}

\section{Walt Patterson}

Energy Policy: Strategies for Uncertainty. By P. Lesley Cook and A. J. Surrey. Pp. 240. (Martin Robertson: London, 1977.) $£ 8.50$.

CONTEMPORARY daredevils have lately come up with two new and hairraising ways to tempt fate. One is skateboarding. The other is energy policy. Both activities can induce in their participants a brisk and delicious exhilaration. Both, however, bring with them the possibility of coming an abrupt and messy cropper. It is undoubtedly desirable to don protective apparel, but no amount of protection can guarantee immunity from disaster.

Lesley Cook and John Surrey have no advice for skateboarders. But they have written a brilliant and vivid depiction of the pitfalls gaping before those intrepid souls who would venture on to the sweeping contours of energy policy. They begin by delineating the background which has given rise to the preoccupation in the 1970s with what is now called 'energy policy'. Here as throughout the book they focus primarily on the UK; but even that constraint in no way leaves them short of monitory historical examples to set the stage. They date the prehistory of 'energy policy' to the White Paper of 1967 on Fuel Policy, which might have been written by Etruscans, so remote are its assumptions from those now coming to the fore.

The subtitle of the book is Strategies for Uncertainty. Chapter 3 identifies and disaggregates many general categories of uncertainty which now confront planners. Part II (chapters 4-9) dissects in detail the overall present-day UK position, and the particulars affecting oil, coal, gas, and electricity, and their interdependence. After the first 149 pages no reader can programme in current prices, such as Professor David Henderson has published.

I have concentrated on illustrating features of the book which in my view limit its value in analysing the problems of organisation and Ministerial intervention and assessing the gains so far from nuclear programmes. I would add in conclusion that I have found it attractive, stimulating rather than provocative, and admirably broad in scope. It deals well with many topics outside the programmes -the Windscale incident, nuclear ship propulsion, growth of safety organisation, 'waste' and the environment, for example; and its account of the consortia is particularly good and, with good judgment, more appreciative than is usual.

Duncan Burn was Prufessor of Economics at the University of Manchester from 1967-69.

have any doubt about the quantity and variety of uncertainty prevailing.

About problems of forecasting, planning of investment, establishment of performance criteria, and coordination of policies between supply industries, Cook and Surrey are incisive and unsparing. It is a pleasure to report that, although both authors are economists, they write crisp and readable prose, commendably free of jargon and accessible to any reader with a modicum of interest in the subject. Indeed any reader who comes to the book without previous involvement may well find the book a stimulus to further interest, because the authors obviously care about the issues they are discussing. Their generalisations are almost invariably drawn from specific-often recent-examples, giving an immediacy to what might in other hands have been a bewildering academic exercise.

Cook and Surrey pinpoint so many full-fledged problems that it is almost sadistic to ask them to note still more. Unfortunately, however, the 'uncertainty' of Part II is a great deal more convincing than the 'strategies' they propose in Part III. Their analysis in Part II, although by far the most comprehensive yet presented, nevertheless falls short. In doing so it undermines Part III. The difficulty arises because Cook and Surrey, despite their perspicacity, approach the energy question almost exclusively from the viewpoint of those responsible for the supply and distribution of fuel. What they are discussing is accordingly not 'energy policy' but fuel policy. That is, to be sure, a necessary and important area for study. But it is not coextensive with 'energy policy', as energy policy is now coming to be understood. The drawback is especially evident as it affects the commendable proposal by Cook and Surrey that future planning should start with a long-term strategy, and then adopt medium- and short-term strategies in keeping with the long-term. It is not, however, possible to develop a long-term strategy without taking far more account of the ways in which fuel energy is used, and of the policy measures which affect its use.

Recent work in the UK and elsewhere has begun to build a data base about the use of energy-domestic, commercial, industrial, transport; about the temperature spectrum of energy requirements; about the time variations of uses; about the technical improvements by which the same end-uses can be accomplished with far more elegance and economy of means; and about the institutional and policy measures which may be implemented to bring about an entirely new approach to the role of fuel energy in society. To mention but one example, the Energy Project of the International Institute for Environment and Development has prepared detailed, disaggregated and costed scenarios which suggest that UK primary fuel energy use in 2025 need be no more than half that in 1978, while standards of personal comfort, mobility and industrial productivity are higher than those of today. This and other similar analyses are now being given serious consideration by the Energy Technology Support Unit of the UK Government Department of Energy. Until such possibilities are incorporated in 'energy policy', alongside considerations affecting investment and planning for additional supply, any 'energy policy' can only be partial. From the point of view of social objectives and values, such a partial policy will be inevitably far from 'optimal', and almost certainly unnecessarily vulnerable; this would include the policy approach proposed here by Cook and Surrey.

In fairness, however, it should be noted that one of the most active and imaginative contributors to the new ferment of innovative energy policy thinking is the Science Policy Research Unit (SPRU) at the University of Sussex, of which John Surrey is a Senior Fellow. SPRU's work on energy use, and disaggregated forecasting, has been a major factor in the gradual enlightenment now dawning at the Department of Energy, and demonstrated-if as yet somewhat tentatively in the recent Green Paper on Energy Policy.

Cook and Surrey completed most of the writing of their book before the end of 1976. They can hardly be faulted if it has by now been slightly overtaken by the unravelling 'uncertainty' which they have foretold.

Indeed, if 'energy policy' is-as seems likely-soon to embrace building standards, transport systems, industrial production and processes, agriculture and land-use planning, and a host of other policy areas, it may before long have outlived its fascination as an identifiable activity. Anyone for skateboarding?

Walt Patterson is Energy Consultant to Friends of the Earth (London) Ltd. 